\title{
Parametric Design in Timber Gridshell Tectonics
}

\author{
Syarifah Ismailiyah Al Athas ${ }^{1, *}$ \\ ${ }^{1}$ Universitas Islam Indonesia, Indonesia, Indonesia
}

\begin{abstract}
This paper begins with a simple proposition: rather than mimicking the geometric structures found in nature, perhaps the most effective modes of sustainable fabrication can be found throughunderstanding the nature of materials themselves. The material becomes a design parameter through the constraints of fabrication tools, limitations of material size, and most importantly the productivecapacity of material resistance a given material's capacity and tendencies to take shape, rather than cutting shape out of material. Gridshell structures provide an intriguing case study to pursue this proposition. Not only is there clear precedent in the form finding experiments of frei Otto and the institute for lightweight structures, but also the very nurbs based tools of current design practices developed from the ability of wood to bend. Taking the bent wood spline quite literally, gridshells provide a means that is at once formally expressive, structurally optimized, materially efficient, and quite simply a delight to experience. The the larger motivation of this work anticipates a parametric system linking the intrinsic material values of the gridshell tectonic with extrinsic criteria such as programmatic needs and environmental response. Through an applied case study of gridshells, the play between form and material is tested out through the author's own experimentation with gridshells and the pedagogical results of two gridshell studios. The goal of this research is to establish a give and take relationship between top down formal emphasis and a bottom-up material influence.
\end{abstract}

Keywords: parametric design, timber construction, gridshell construction

\section{Introduction}

The tension between form and material is neither a new one, nor is it necessarily a digital matter. As early as 1934, henri focillon suggests that matter imposes its own form upon form (focillon 1934). With today's digital fabrication tools, material is all too often seen as a homogenous substrate with the outputs of digital fabrication tools essentially creating physical representations of digitally derived form. Useful and at times inspiring, yet clearly this matter does not impose its own form upon form. Said another way, there is not an inherent material feedback loop in the digital design process.

Material morphogenesis, as the name would suggest, is more than simply a material feedback loop in the design process, but suggests a material system that derives form. Contemporary fabrication tools are not simply about material output, but are a means to extend the material capacity, complexity, and variation of a material system. Research practices such as ocean north are a noticeable proponent of this approach. They note that "natural morphogenesis" develops its complex form from the systematic interaction between internal material capacities and external environmental influences and forces. In- spired from this natural morphogenesis, they situate their "computational morphogenesis" on the complex interrelationships between material capacities, manufacturing constraints and logics of assembly, and external micro-climatic conditions. In achim menges' teaching research at the architectural association's design research lab (aadrl), focus is placed on micro systems on material assemblies and their aggregate effect on larger structures. They emphasize a bottom-up approach, critiquing the "indulgence" of 1990's formalism (menges 2007).

\section{Literature Review}

The potentials for a bottom-up, material centric design approach are far more than sim- ply pragmatic issues, but have strong philosophical, conceptual, and perhaps even ethical values. Working backwards through a chain of philosophical influences from gilles deleuze and felix guattari to henri bergson, manual delanda, like focillon, is working against the philosophical indifference to material. This platonic indifference suggests that ideas are simply actualized by the addition of matter - a view that matter is an inert receptacle of ex- ternal ideas. Rather, the view delanda takes up is that material is an active participant in the genesis of form. He focuses on two central aspects of material qualities: their capacity and tendencies (delanda 2004). To oversimplify this case in the wood spline, its capaci- ties are its bending strength and its tendencies develop from its axis in bending (bi-axial vs. Uni-axial). These material capacities and tendencies are material constraints. From a material morphogenetic point of view, constraints are not simply limitations, but become pro- ductive by shaping design parameters. Quite literally, material constraints ground design. Viewing constraints as productive can have a powerful affect on design concepts. After all, attentiveness to materials directs ones attention beyond the symbolic and representational nature of design and instead prioritizes the

* Corresponding author: syarifah.alathas@uii.ac.id 
environmental, kinesthetic and haptic experi- ence of architecture. Consequently, the primary visual interface of digital media may be balanced by the material influence through taking the wood spline quite literally. Through this, there is a connection between material values and digital parameters. All too often the "parameters" of digitally derived work are abstract points - if abstracted at all-from which form is instrumentalized. These 'datascapes,' as reiser + umemoto contend, are "an un- fortunate consequence of design in the semantic mode" (reiser 2006: 217). Suggesting that values and parameters are not, in the end, synonymous, provides an opportunity to productively connect the two rather than conflate them as one and the same thing. While many may argue that the free-form fascination in contemporary architecture is a consequence of nurbs based software, an overlooked historical fact is that material constraints and parametric flexibility were the foundations of the system. While pierre bezier, among many other mathematician/engineers, is the father of today's nurbs systems, the motivation to develop the system was based on a coupling between a simple parametric structure in response to the physical constraints of computer-aided manufacture. One year before his death, pierre bezier recounts: "to sum up the basic ideas of the system, it can be said that it came from the ability to work, think, and react in the rigid cartesian world of machine tools and, at the same time, in the more flexible, ndimensional parametric world" (bezier 1998). Of course, the very physical spline that bezier abstracted into what we now know of as the bezier curve, contained both the material resistance of the wood spline and the geometric constraints of the weighted ducks. Ironically, in abstracting the geometry of the constraints, the materials capacities and tendencies were abstracted out of the sys- tem. Simply put, material constraints are not built into this digital system. In exchange for these lack of material constraints, and the restriction to plane curves ( $2 \mathrm{~d}$ curves) and fixed spline lengths, we get an incredibly adaptable flexible system built upon space curves ( $3 \mathrm{~d}$ curves) and infinitely extensible curves. In architecture this has given a new formal renais- sance, and the blob as a new typology. However, the accessibility of nurbs based software long before the accessibility of today's digital fabrication tools has only extended the philosophical ideas that material is a receptacle of externally driven forms. Consequently, taming this wild geometry is so frequently done through slicing and egg crating that these techniques have become synonymous with digital fabrication.

This research takes the material spline quite literally by looking at its capacity to take shape, rather than cutting shape out of material, as well as the tendencies of the cross-sectional area of the spline to bend in a very constrained manner. While clearly a conservative and very constrained approach, the result is a give-and-take relationship between material and geometry that is easily constructible, materially efficient, and structurally expressive architecture. In the end, the goal is not to suggest gridshells are the answer, but rather, that the precedent of gridshells may be a pedagogical tool developing material values in a digi- tal design culture.

\section{Gridshell Precedent}

The first examples of gridshells were developed through the partnership of architect frei otto and a young ted happold, then at ove arup, exemplified by the timber gridshell at mannheim in germany built in 1975. Although very few gridshells have been built, there has been a resurgence of these structures in the last eight years through shigeru ban's japanese pavilion and jacques herzog's expodach for expo 2000 in hanover, helsinki university of technology wood studio's timber bubble at the helsinki zoo (2003), edward cullinan's downland gridshell (2002), and most recently glenhowells architects' savill building (2006). Typically these structures are fabricated from a flat mat of straight laths, or paper tubes in ban's pavillion, and then raised and/or lowered into shape. The expodach is a prefabricated lamella approach and the timber bubble is constructed piece by piece of preformed laminated laths.

Structurally, gridshells have the properties of a structural shell, which gains its strength and stiffness through curvature, with a shell formed from double curvature as the most ef- ficient in terms of minimum use of material. Gridshells are typically constructed through a criss-crossed pattern of straight laths. Synclastic surfaces are curved only in tension or only in compression. For example, an arch is formed using only compression, and a dome is a rotated arch. Geodesic domes are examples of synclastic shapes. Anticlastic surfaces, or saddle shapes, have tension forces in one direction and compressive forces in the other stabilized by the tension forces. The significance of anticlastic surfaces is that they are more flexible in their formal morphology and structurally more efficient from the balance between tension and compression forces.

\section{Gridshells Applied}

This applied gridshell research developed over the course of about one year and included teaching two gridshell studios. Presented first are the techniques and challenges of developing gridshells with conventional digital tools, and then briefly presented are the varied approaches taken in studio work. Despite the curving surfaces of gridshells, they are typi- cally constrained to straight barrel vault warehouse like spaces as a result of the flat matt technique of raising or lower lattice gridshells. To challenge this plan-oriented constraint, the base surface for this experiment developed from a 90degree turn through four bound- ary curves and one center curve.

\section{Lath Pattern: Projected, Applied, Geodesic}

A pattern can be projected onto a surface, however this is limited as the pattern can only be projected in one direction. Another method is to simply "apply curves" to a nurbs surface. While at first it appears this is an easy method to depart from the opposing uv logic of nurbs surfaces, in fact, the pattern is stretched proportionally across the surface in because each node is located at the surface normal, the node connection is square to the flattened unrolled lathe. Consequently, when the laths are put in place, the node holes 
will align only when the laths take their correct position as a kind of pin registration. Through a trial and error process, the applied grid pattern was developed such that an unrolled lath could fit on a $2 \times 8$ board of clear oregon pine. A section model was built as a proto- type testing the accuracy of these developed surfaces and the pin registration at the nodal points (figures six and seven). While the model was successful and easily assembled, cut- ting crescent shapes from solid boards of clear oregon pine, only utilizing about $60 \%$ of the material, was a contradiction in the goals of the project to do more with less.

\section{Surface Relaxation: Form and Geodesic Pattern Revisited}

In the form-finding procedures of frei otto, the hanging chain models find a minimum en- ergy form - their relaxed state. In contemporary examples such as the downland gridshell, a software based dynamic relaxation technique was employed. Connecting the minimum energy geodesic curve with a minimum energy (relaxed) surface seamed a plausible ap- proach. Through a surface relaxation plug-in (www.reconstructivism.net), the base surface was relaxed with the four boundary curves constrained. The grid pattern was applied, and from these same endpoints a geodesic net was developed.

In the relaxed surface, a geodesic net is evenly distributed, and was therefore able to be developed from straight laths. This simple example proves a signifi- cant point: a give-and-take relationship between top-down formal emphasis and a bottom- up material influence is necessary.

\section{Gridshell Studios}

The gridshell studios were given as a context to teach a bottom-up materials first approach in developing complex form. While the relevance of this studio was conceived at a school that was digital savvy, the studios were actually taught at two different schools with upper

Division students who had very marginal, if any, digital modeling skills. The studios began through graphic case studies of built gridshells, as well as building physical prototypes of these case studies to feel the forces at play in these structures. Physically testing material to its limits was of interest to these students, yet their digital skills lacked the geometric attention that the above approaches necessitated. From these case studies, the students were welcome to critique the gridshell's appropriateness for this project, as well as to de- velop new approaches to gridshell inspired designs. Four approaches are illustrated here.

\section{Gridshell Mold}

The most conservative though straightforward approach was to develop a $3 \mathrm{~d}$ mold and then apply laths individually over the mold. This was the first time for this graduate student to use a cnc router, and he was noticeably excited to see this surface emerge from a block of foam. After seeing and touching this surface for flat spots, he quickly located areas for revision, though regretfully he felt it was too late in the term to alter and re-mill his mold. He then meticulously glued a four-layer lath over the mold using t-pins to hold the laths in place.

An alternate mold approach, inspired by the prefabricated shells of the expodach, em- ployed a modular mold using overlapping rings instead of straight laths. The ring pattern was applied to the surface and unrolled as developable surfaces. Each ring was laminated from two paper strips to hold their circular shape, and then connected at the quadrant points through overlapping bridal joints.

\section{Standardized Unit / Variable Shim Approach}

One team greatly resisted that each piece would be unique and pursued an approach that used a standardized unit with the space between the units providing the variation. A series of laser cut boxes with variable shims between the boxes was used to physically model the various configurations this approach could take. Ironically this straight- forward approach is exceedingly difficult to digitally model with conventional tools. While their digital representations were approximations of this approach, they also built a full- scale mock-up to test this modular approach.

\section{Periodic Laminated Approach}

Perhaps the most sophisticated approach was developed through a periodic surface and a hexagonal tessellated pattern. Lacking sophisticated skills and tools to track a series of unique pieces, the periodic surface and pattern configuration created a unique, two directional, waveform from only 12 unique pieces. Like the prefabricated lamella structure of the expodach, prefabricating a modular lamella gridshell surface over these larger spans could attain further enclosure.

\section{Conclusion}

The intent of this applied research is to propose an economy of means through under- standing the nature of materials rather than mimicking the aesthetics of natural systems. While digital fabrication tools are a welcome and significant addition to the architects' tool- set, the 'digital' aspect of these tools typically suggest form first only much later to con- sider material, if at all. Gridshells inverse this relationship suggesting a bottomup mate- rials first approach to form finding. However, the matt technique of developing gridshells yields a very constrained formal morphology, and while elegant, has limited application. The gridshell tectonic developed here intends to balance the geometric constraint based on a physical laths capacity and tendencies to take shape with a more globally flexible form suggesting a giveand-take relationship between a bottom- up and topdown formal orga- nization. It also serves to critique the proportional distribution of surface sub-division and 
applied patterns in parametric tools which are still tied to the non-material nurbs surface, in favor of approaches that use geodesic or minimal energy curves derived from a materi- als capacities and tendencies to balance form and material.

\section{References}

Achim, Menges. (2007). “Computational Morphogenesis: Integral Form Generation And Materialization Processes," Proceedings Of The 3rd International Ascaad Conference On Em'body'ing Virtual Architecture.

Bezier, Pierre. (1998). "A View Of The Cad/Cam Development Period," Ieee Annals Of The History Of Computing 20 (2): 37-40.

Delanda, Manuel. (2004). "Material Complexity," In Neil Leach, David Turnbull, And Chris Williams, Digital Tectonics. London: Wiley-Academy Press.

Focillon, Henri. (1934/1992). The Life Of Forms In Art. New York: Zone Books.

Harris, Richard, John Romer, Oliver Kelly, And Stephen Johnson. "Design And Construction Of The Downland Gridshell," Building Research And Information 6 (31): 427-454.

Reiser, Jesse. (2006). Atlas Of Novel Tectonics. New York: Princeton Architectural Press. 